\title{
Medium effects in the nuclear Landau-Vlassov transport theory
}

\author{
J. Cugnon and A. Lejeune \\ Physique Nucléaire Théorique, Institut de Physique au Sart Tilman, Université de Liege, \\ B-4000 Liege 1, Belgium \\ P. Grangé \\ Centre de Recherches Nucléaires, F-67037 Strasbourg Cedex, France
}

(Received 23 September 1986)

\begin{abstract}
With the assumption that the Brueckner $g$ matrix provides the suitable renormalization of the nucleon-nucleon interaction due to medium effects in the collision term of the nuclear LandauVlassov equation, we use the results of our Brueckner calculation for nuclear matter at finite temperature to evaluate the renormalization factor in a local and thermal approximation.
\end{abstract}

In the last months, there has been an intensive search for a suitable (and still tractable) transport equation for heavy ion collisions in the so-called intermediate energy regime, ${ }^{1-3}$ following a similar effort in the high-energy domain. ${ }^{4-6}$ Apparently, a rather large consensus emerges on the fact that such an equation should have a Landau-Vlassov (LV) structure:

$$
\left(\frac{\partial}{\partial t}+\mathbf{p} \cdot \mathbf{\nabla}-(\boldsymbol{\nabla} U) \cdot \nabla_{\mathbf{p}}\right) f(\mathbf{r}, \mathbf{p}, t)=G-L,
$$

with $(\hbar=1)$

$G=\int \frac{d^{3} p_{2}}{(2 \pi)^{3}} \frac{d^{3} p_{3}}{(2 \pi)^{3}} \frac{d^{3} p_{4}}{(2 \pi)^{3}} \omega\left(p p_{2} \leftarrow p_{3} p_{4}\right) f_{3} f_{4}(1-f)\left(1-f_{2}\right) \delta^{3}\left(\mathbf{p}+\mathbf{p}_{2}-\mathbf{p}_{3}-\mathbf{p}_{4}\right) \delta\left[e(p)+e\left(p_{2}\right)-e\left(p_{3}\right)-e\left(p_{4}\right)\right]$

and

$L=\int \frac{d^{3} p_{2}}{(2 \pi)^{3}} \frac{d^{3} p_{3}}{(2 \pi)^{3}} \frac{d^{3} p_{4}}{(2 \pi)^{3}} \omega\left(p p_{2} \rightarrow p_{3} p_{4}\right) f f_{2}\left(1-f_{3}\right)\left(1-f_{4}\right) \delta^{3}\left(\mathbf{p}+\mathbf{p}_{2}-\mathbf{p}_{3}-\mathbf{p}_{4}\right) \delta\left[e(p)+e\left(p_{2}\right)-e\left(p_{3}\right)-e\left(p_{4}\right)\right]$

[Equation (1), however, presents some weak points, the most important of which is the lack of memory effects in the collision terms. This has the bad consequence of unphysically small damping in some particular situations. $\left.{ }^{7}\right]$ In this equation, $f(\mathbf{r}, \mathbf{p}, t)$ can be considered as the probability of finding a particle with momentum $\mathbf{p}$ at position $\mathbf{r}$ (or at least as the usual quantal Wigner function) normalized as

$$
\iint \frac{d^{3} \mathbf{p} d^{3} \mathbf{r}}{(2 \pi)^{3}} f(\mathbf{r}, \mathbf{p}, t)=A,
$$

where $A$ is the mass number of the system, $U$ is the average single particle field, $\omega\left(\mathbf{p p}_{2} \rightarrow \mathbf{p}_{3} \mathbf{p}_{4}\right)$ is the transition probability for a collision between two particles of momenta $\mathbf{p}$ and $\mathbf{p}_{2}$ to give a final state with momenta $\mathbf{p}_{3}$ and $\mathbf{p}_{\mathbf{4}}$, and $f_{i}$ stands symbolically for $f\left(\mathbf{r}, \mathbf{p}_{i}, t\right)$. Finally, $e(p)$ is the single-particle energy.

The situation is, however, confused concerning what is the exact meaning of the "input" quantities of Eq. (1), and, especially of the transition matrix $\omega$. The theoretical basis for the LV equation has been studied by several authors. ${ }^{4,7-11}$ In general, the LV equation is obtained in the weak coupling limit, for fermions interacting through potentials $v$. In that case

$$
\omega\left(p p_{2} \rightarrow p_{3} p_{4}\right)=\left|\left\langle\mathbf{p}_{2}|v| \mathbf{p}_{3} \mathbf{p}_{4}\right\rangle\right|^{2} .
$$

In nuclear physics, the interaction is too strong for this limit to be valid. Rather, it is considered that the free transition matrix

$$
\omega\left(p p_{2} \rightarrow p_{3} p_{4}\right)=\left|\left\langle\mathrm{pp}_{2}|T| \mathbf{p}_{3} \mathrm{p}_{4}\right\rangle\right|^{2}
$$

would be a more appropriate choice. The state vectors in Eq. (6) are referring to free plane waves. But, medium corrections which are well studied in equilibrium situations (for a review, see Ref. 12, e.g.) are also expected in not too far from equilibrium situations, like in medium-energy heavy ion collisions. Recently, Botermans and Malfliet ${ }^{13}$ have shown that the usual Brueckner resummation of ladder diagrams can be made in very much the same way as in equilibrium situations. Therefore, the most reasonable choice seems to be

$$
\omega\left(p p_{2} \rightarrow p_{3} p_{4}\right)=\left|\left\langle\mathbf{p p}_{2}|g| \mathbf{p}_{3} \mathbf{p}_{4}\right\rangle\right|^{2},
$$

where $g$ is the usual Brueckner reaction matrix, describing the scattering of two nucleons in a nuclear medium. In principle, $g$ should be recalculated for the instantaneous occupation in phase space, described by $f(\mathbf{r}, \mathbf{p}, t)$. However, this is a tremendous task which can be avoided if the momentum distribution can be approximated by a FermiDirac distribution at temperature $T$, which seems reasonable for not too far from equilibrium situations. Indeed, 
for these conditions, the $g$ matrix has been calculated for several densities and temperatures, typical of the medium energy domain.

Here, we use our recent calculation ${ }^{14}$ to present the factors by which the collision term with the free transition matrix [Eq. (6)] should be multiplied in order to incorporate medium corrections. In the conditions described above, the loss term reads

$$
\begin{aligned}
L=f \int & \frac{d^{3} p_{2}}{(2 \pi)^{3}} \frac{d^{3} p_{3}}{(2 \pi)^{3}} \frac{d^{3} p_{4}}{(2 \pi)^{3}} f_{2}\left(1-f_{3}\right)\left(1-f_{4}\right)\left|\left\langle\mathbf{p} \mathbf{p}_{2}|g(\rho, T)| \mathbf{p}_{3} \mathbf{p}_{4}\right\rangle\right|^{2} \\
& \times \delta^{3}\left(\mathbf{p}+\mathbf{p}_{2}-\mathbf{p}_{3}-\mathbf{p}_{4}\right) \delta\left[e(p)+e\left(p_{2}\right)-e\left(p_{3}\right)-e\left(p_{4}\right)\right]
\end{aligned}
$$

where $\rho$ is the local baryon density. It is interesting to note that Eq. (8) can be rewritten as ${ }^{12}$

$$
L=\frac{2}{\pi} f W(p, \rho, T),
$$

where $W$ is the imaginary part of the optical-model potential (evaluated in second order in $g$ ) for a particle of momentum $p$ traveling in a medium of density $\rho$ at temperature $T$. We will denote by $\alpha$ the ratio

$$
\alpha=\frac{W(p, \rho, T)}{\tilde{W}(p, \rho, T)},
$$

where

$$
\tilde{W}(p, \rho, T)=\int \frac{d^{3} p_{2}}{(2 \pi)^{3}} \int \frac{d^{3} p_{3}}{(2 \pi)^{3}} \int \frac{d^{3} p_{4}}{(2 \pi)^{3}} f_{2}\left(1-f_{3}\right)\left(1-f_{4}\right)\left|\left\langle p p_{2}|T| p_{3} p_{4}\right\rangle\right|^{2} \delta^{3}(\mathbf{p}) \delta\left(\frac{p^{2}}{2 m}+\frac{p_{2}^{2}}{2 m}-\frac{p_{3}^{2}}{2 m}-\frac{p_{4}^{2}}{2 m}\right)
$$

is the value the imaginary part would take if the interaction were not influenced by the medium. It is clear that medium effects are operating in two places. (i) The phase-space is distorted because the particles are feeling a mean field, which is embodied by the energy-conserving $\delta$ function in Eq. (8). (ii) The interaction itself is modified, which is responsible for the replacement of the $T$ matrix

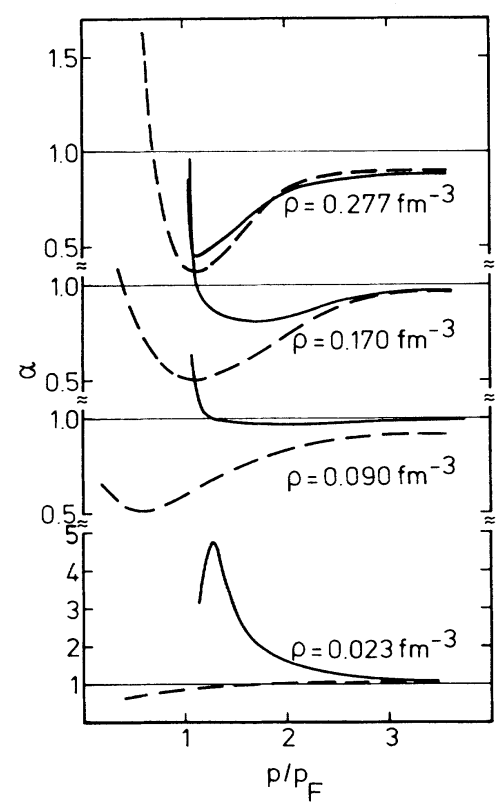

FIG. 1. Renormalization factor $\alpha(p, \rho, T)$ correcting for medium effects [Eq. (10)], for several densities and for two temperatures: $T=0$ (full curves) and $T=10 \mathrm{MeV}$ (dashed curves). The quantity $\alpha$ is given as a function of the momentum $p$. The quantity $p_{F}$ is the usual Fermi momentum for the considered density. by the $g$ matrix. This modification comes in turn from two effects, as is well known. (1) The Pauli principle forbids having intermediate states that are already occupied. (2) The particles are feeling the mean field in the intermediate states.

It is clear from Eq. (10) that $\alpha$ is the required multiplicative factor that accounts for medium corrections in the loss term (in the conditions mentioned above) compared to the commonly used Eq. (11). We have extracted the numerical values of $W(p, \rho, T)$ from our Breuckner calculation. $^{14}$ The quantity $\tilde{W}$ has been calculated by Eq. (11) with the use of Fermi-Dirac distributions. In order to simplify the numerical task, we have used the so-called angleaveraged Pauli operator, which amounts to replacing $\left(1-f_{3}\right)\left(1-f_{4}\right)$ by its average over the angles between $\mathbf{p}_{3}$ and $\mathbf{p}_{4}$. This approximation is quite sufficient for our purpose here and will be discussed extensively in a separate publication. In that case, the integration over one of the variables yields the integrated nucleon-nucleon cross section, as it is well known. ${ }^{15}$ We have used the phenomenological values of Ref. 16.

TABLE I. Coefficients entering in the representation of the factor $\alpha$ [see Eq. (16)], in $\mathrm{fm}^{j}$ units, for two temperatures.

\begin{tabular}{ccccc}
\hline \hline$a_{n j}$ & $j=1$ & $\begin{array}{c}j=2 \\
T=0\end{array}$ & $j=3$ & $j=4$ \\
& & 19.655 & 99.017 & -140.75 \\
$n=1$ & -15.560 & 417.00 & -1437.10 & 1268.8 \\
$n=2$ & -14.896 & -1754.9 & 3919.8 & -2795.9 \\
$n=3$ & 253.26 & $T=10$ & & \\
& & -69.733 & 103.54 & -41.842 \\
& & 104.86 & -156.78 & 54.296 \\
$n=1$ & 13.966 & -50.211 & 78.582 & -26.551 \\
$n=3$ & 9.8803 & & & \\
\hline \hline
\end{tabular}


Our results are contained in Fig. 1. There is a considerable correction at small density, especially for cold matter. Surprisingly, the renormalization at $\rho=0.090 \mathrm{fm}^{-3}$ and $T=0$ is small, but this is accidental, as we shall see below. For ordinary density and for $\rho=0.277 \mathrm{fm}^{-3}$, the renormalization factor $\alpha$ is essentially below unity. As one can see, the temperature dependence is less and less important as the density increases. At $\rho=0.277 \mathrm{fm}^{-3}$, it is almost negligible, except, of course, that at $T \neq 0$ a nucleon with momentum less than the Fermi momentum $k_{F}$ can induce real transitions. The medium correction may enhance

(essentially at low density) or reduce (at high density) the effective scattering cross section, and therefore the loss term, contrary to the Pauli blocking factor $\left(1-f_{3}\right)\left(1-f_{4}\right)$ which always reduces the scattering probability in the conditions studied here, as can be deduced from the results of Ref. 17. To make the comparison with the latter work clearer, we denote by $\alpha^{(0)}$ the following ratio:

$$
\alpha^{(0)}=\frac{\tilde{W}(p, \rho, T)}{W^{N P}(p, \rho, T)},
$$

$W^{N P}(p, \rho, T)=\int \frac{d^{3} p_{2}}{(2 \pi)^{3}} \int \frac{d^{3} p_{3}}{(2 \pi)^{3}} \int \frac{d^{3} p_{4}}{(2 \pi)^{3}} f_{2}\left|\left\langle\mathbf{p p}_{2}|T| \mathbf{p}_{3} \mathbf{p}_{4}\right\rangle\right|^{2} \delta^{3}\left(\mathbf{p}+\mathbf{p}_{2}-\mathbf{p}_{3}-\mathbf{p}_{4}\right) \delta\left(\frac{p^{2}}{2 m}+\frac{p_{2}^{2}}{2 m}-\frac{p_{3}^{2}}{2 m}-\frac{p_{4}^{2}}{2 m}\right)$.

As we said, this ratio is always smaller than unity, and in Ref. 17 , the quantity $\alpha^{(1)}=\alpha \alpha^{(0)}$ is actually calculated.

In order to present our results in a convenient form, we have fitted the function $\alpha$ by the following algebraic form:

$$
\alpha(p, \rho, T)=1+\sum_{n=1}^{3} \sum_{j=1}^{4} a_{n j}(T) \frac{\rho^{j / 3}}{\left(p / p_{F}\right)^{n}},
$$

where the coefficients $a_{n j}$ are given in Table I for $T=0$ and $T=10 \mathrm{MeV}$. The fit is of very good quality, except close to $p=p_{F}$. But in that particular case, the loss term is very small anyway. In general, the loss term is small for $k \approx k_{F}$ and decreases with decreasing density.

The medium effect on the single-particle energies can be incorporated readily in the collision term. The "free scattering" loss term would then be given by Eq. (9) with the following expression of the optical-model potential:

$\bar{W}(p, \rho, T)=\int \frac{d^{3} p_{2}}{(2 \pi)^{3}} \frac{d^{3} p_{3}}{(2 \pi)^{3}} \frac{d^{3} p_{4}}{(2 \pi)^{3}} f_{2}\left(1-f_{3}\right)\left(1-f_{4}\right)\left|\left\langle\mathbf{p p}_{2}|T| \mathbf{p}_{3} \mathbf{p}_{4}\right\rangle\right|^{2} \delta^{3}(\mathbf{p}) \delta\left[e(p)+e\left(p_{2}\right)-e\left(p_{3}\right)-e\left(p_{4}\right)\right]$,

instead of Eq. (11). In the simplest case, the singleparticle energies can be taken in the constant effective mass approximation

$$
e(p)=\frac{p^{2}}{2 m^{*}}+U_{0},
$$

where $U_{0}$ is a constant. The quantity $m^{*}$ is extracted from Ref. 14 for the same conditions of density and temperature. Its value is given in Table II.

The ratio of $W$ to $\bar{W}$,

$$
\alpha^{\prime}=\frac{W(p, \rho, T)}{\bar{W}(p, \rho, T)}
$$

then really represents the importance of the medium renormalization of the scattering of two nucleons, weighted by the appropriate kinematical conditions. It is given in Fig. 2 for illustration. This renormalization coefficient may also lie above or below unity.

Note that, due to the approximation (16) with a constant $m^{*}$, the ratio $\alpha^{\prime}$ has not the proper asymptotic limit for large $k$. However, it is known that in a realistic calculation, $m^{*}$ comes close to unity for larger values of $k$ than those considered in Fig. 2.

TABLE II. $m^{*} / m$ values.

\begin{tabular}{cllll}
\hline \hline & \multicolumn{4}{c}{$\rho\left(\mathrm{fm}^{-3}\right)$} \\
$T(\mathrm{MeV})$ & 0.023 & 0.09 & 0.17 & 0.28 \\
\hline 0 & 0.853 & 0.725 & 0.733 & 0.671 \\
10 & 0.787 & 0.770 & 0.778 & 0.752 \\
\hline \hline
\end{tabular}

In the conditions considered above, i.e., in the thermal equilibrium limit, the gain term is equal to the loss term. Since this is independent of the transition probability, the same renormalization has to be applied to the gain term. This renormalization is presumably not very much dif-

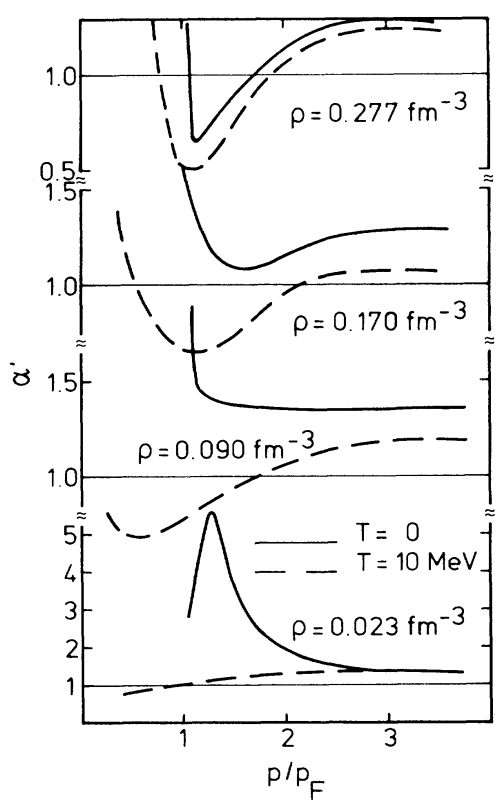
(17)].

FIG. 2. Same as Fig. 1 for the factor $\alpha^{\prime}$ [see text and Eq. 
ferent if one departs from equilibrium. Let us remind ourselves that in the intermediate energy domain, the system is never far from equilibrium. This is confirmed by the fact that the matter, if totally thermalized would reach temperatures of $10 \mathrm{MeV}$ or less.

In conclusion, we have calculated, using the local density approximation, the medium renormalization of the collision term in a LV-type transport equation. In the density and temperature domain studied here, which is typical of the medium-energy heavy ion collision, this renormalization is not negligible and may be an important influence on the equilibration process. Let us remind ourselves that the average equilibration time, $\sim 10 \mathrm{fm} / c$, is smaller but not much smaller than the collision time. ${ }^{18}$ Finally, we stress that the renormalization factor can be larger or smaller than unity, depending upon the kinematical conditions.

We are grateful to Dr. H. S. Köhler for an interesting correspondence and to the Fonds National de la Recherche Scientific, Belgium, for the opportunity to use a CRAYXMP computer. Two of us (J.C. and A.L.) would like to thank very much the Centre de Recherche Nucleaires, Strasbourg, for the kind hospitality extended to them. This work was supported by NATO Research Grant No. 025.81 .
${ }^{1} \mathrm{~J}$. Aichelin and G. Bertsch, Phys. Rev. C 31, 1730 (1985).

${ }^{2}$ C. Grégoire, B. Remaud, F. Scheuter, and F. Sébille, Nucl. Phys. A436, 365 (1985).

${ }^{3}$ H. S. Köhler, Nucl. Phys. A343, 315 (1980); A440, 165 (1985).

${ }^{4}$ P. Danielewicz, Ann. Phys. (N.Y.) 152, 239 (1984); 152, 305 (1984).

${ }^{5}$ G. Bertsch, H. Kruse, and S. Gupta, Phys. Rev. C 29, 673 (1984).

6J. Molitoris, J. B. Hoffer, H. Kruse, and H. Stöcker, Phys. Rev. Lett. 53, 899 (1984).

${ }^{7}$ P. Grangé, H. A. Weidenmüller, and G.Wolschin, Ann. Phys. (N.Y.) 136, 190 (1981); R. Balian and M. Vénéroni, ibid. 135, 270 (1981); P. Grangé, J. Richert, G. Wolschin, and H. A. Weidenmüller, Nucl. Phys. A356, 260 (1981).

${ }^{8}$ J. M. Luttinger and P. Nozières, Phys. Rev. 127, 1431 (1962).

${ }^{9}$ L. P. Kadanoff and G. Baym, Quantum Statistical Mechanics
(Benjamin, New York, 1962).

${ }^{10} \mathrm{P}$. Wolfle, Z. Phys. 232, 38 (1970).

${ }^{11}$ H. H. K. Tang and P. J. Siemens, Nucl. Phys. A453, 251 (1986).

12 J.-P. Jeukenne, A. Lejeune, and C. Mahaux, Phys. Rep. 25C, 85 (1976).

${ }^{13}$ W. Botermans and R. Malfliet, Phys. Lett. 171B, 22 (1986).

${ }^{14}$ A. Lejeune, P. Grangé, M. Martzolff, and J. Cugnon, Nucl. Phys. A453, 189 (1986).

${ }^{15}$ K. Kikuchi and M. Kawai, Nuclear Matter and Nuclear Reactions (North-Holland, Amsterdam, 1968), Chap. 2.

${ }^{16}$ N. Metropolis et al., Phys. Rev. 110, 204 (1958).

${ }^{17}$ A. Lejeune, J. Cugnon, and P. Grangé, J. Phys. (Paris) Colloq. 47, C4-373 (1986).

${ }^{18}$ B. Remaud, C. Grégoire, F. Sébille, and L. Vinet, Ganil Report No. P86-15 (unpublished). 